УДК 621.396 .96

\title{
Increased Noise Immunity Method \\ of a Pulse-Doppler Airborne Radar Station \\ under the Influence of DRFM Signal-Like Interference
}

\author{
Alexander V. Bogdanov, \\ Denis V. Zakomoldin* and Sergey A. Chasovskikh \\ Military Academy of Aero-Space Defence \\ named after the Marshal of Soviet Union G.K. Zhukov \\ Tver, Russian Federation
}

Received 02.08.2020, received in revised form 15.08.2020, accepted 15.09.2020

Abstract. In this article an increased noise immunity method of a pulse-doppler airborne radar station under the influence of a DRFM signal-like interference when exposed on the side lobes of antenna directivity chat has been developed, a device for its implementation has been presented, and characteristics of the device based on the modeling have been evaluated.

Keywords: DRFM signal-like interference, pulse-doppler airborne radar station.

Citation: Bogdanov A.V., Zakomoldin D.V., Chasovskikh S.A. Increased noise immunity method of a pulse-doppler airborne radar station under the influence of DRFM signal-like interference, J. Sib. Fed. Univ. Eng. \& Technol., 2020, 13(7), 803-817. DOI: $10.17516 / 1999-494 X-0267$

\section{Метод повышения помехоустойчивости}

импульсно-доплеровской бортовой радиолокационной станции при воздействии сигналоподобной помехи типа DRFM

\author{
А.В. Богданов, Д.В. Закомолдин, С.А. Часовских \\ Военная академия воздушно-космической оборонь \\ им. Маршала Советского Союза Г.К. Жукова \\ Российская Федераџия, Тверь
}

Аннотация. В данной статье разработан метод повышения помехоустойчивости импульснодоплеровской бортовой радиолокационной станции при воздействии сигналоподобной

(C) Siberian Federal University. All rights reserved

This work is licensed under a Creative Commons Attribution-Non Commercial 4.0 International License (CC BY-NC 4.0).

* Corresponding author E-mail address: denjuga68@yandex.ru 
помехи типа DRFM по боковым лепесткам диаграммы направленности антенны, приведено устройство его реализующее, а также оценены характеристики данного устройства на основе моделирования.

Ключевые слова: сигналоподобная помеха типа DRFM, импульсно-доплеровская бортовая РЛС.

Цитирование: Богданов, А.В. Метод повышения помехоустойчивости импульсно-доплеровской бортовой радиолокационной станции при воздействии сигналоподобной помехи типа DRFM / A.B. Богданов, Д.В. Закомолдин, С.А. Часовских // Журн. Сиб. федер. ун-та. Техника и технологии, 2020. 13(7). С. 803-817. DOI: 10.17516/1999-494X-0267

\section{Введение}

Ведущая роль в завоевании превосходства над противником в современных вооруженных конфликтах принадлежит силам авиации. В успешном выполнении задач силами авиации ключевая роль отводится активному применению средств радиоэлектронного подавления в интересах, с одной стороны, снижения эффективности функционирования РЭС противника, а с другой - использования помехозащищенных бортовых радиолокационных станций (БРЛС) для сохранения их возможностей в условиях радиопротиводействия со стороны противника [1-4].

К настоящему времени разработано большое количество как способов радиопротиводействия, так и способов помехозащиты БРЛС [4-15].

Одним из эффективных способов радиоподавления БРЛС является использование сигналоподобных и шумовых прицельных по частоте силовых помех как по основному, так и по боковым лепесткам диаграммы направленности антенны (ДНА), и технологий сверхбыстрых способов постановки этих помех (технология DRFM - цифровая радиочастотная память). Данная технология позволяет пристыковывать помеховый (усиленный и переизлученный зондирующий сигнал) сигнал с запаздыванием от переотраженного зондирующего сигнала на величины порядка $10^{-9} \ldots 10^{-12}$ с при воздействии помехи по основному лепестку ДНА, что в свою очередь существенно усложняет процесс селекции полезного сигнала, а при необходимости воздействия помехи по боковым лепесткам ДНА осуществить запоминание цифровой копии сигнала и переизлучение ее в направлении на подавляемый объект.

К примеру, известно о принципиальной возможности создания шумовых помех по боковым лепесткам ДНА, согласованных по спектру с зондирующим сигналом на основе технологии DRFM при противодействии двух постановщиков активных помех двум истребителям с их БРЛС [8], а также о методах помехоустойчивости БРЛС, которые в общем случае сводятся к двум основным: метод подавления боковых лепестков (ПБЛ) и метод компенсации боковых лепестков (КБЛ) [9]. Физический смысл метода ПБЛ заключается в блокировании дальнейшей обработки принимаемого сигнала при мощном помеховом воздействии по боковым лепесткам ДНА. Возможность практического использования несовершенства данного метода со стороны противника, оснащенного станцией помех, может быть рассмотрена на примере тактической ситуации, представленной на рис. 1 , схематично отображающей процесс постановки силовой прицельной по спектру шумовой помехи [8]. При облучении основным лепестком диаграммы направленности первого постановщика помех, получив такую информацию, второй постановщик помех направляет помеховое воздействие по боковым лепесткам ДНА первого истребителя, в результате чего блокируется дальнейшая обработка полезного сигнала. Аналогично 


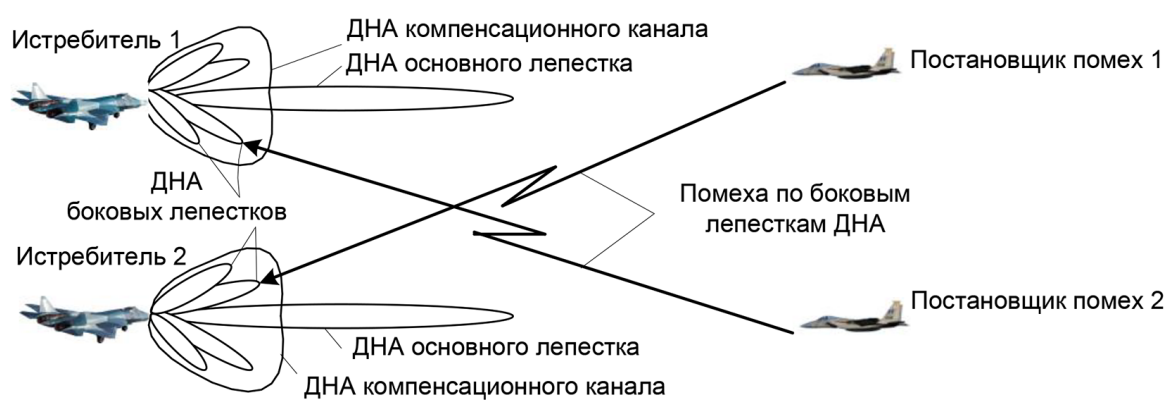

Рис. 1. Схема организации постановки силовой согласованной по спектру шумовой помехи по боковым лепесткам диаграммы направленности антенны

Fig. 1. Scheme organization setting power consistent spectrum noise interference side lobes antenna patters

симметрично действует первый постановщик помех, направляя свой луч помехи на второй истребитель. Если оба истребителя направляют основной лепесток ДНА своих БРЛС на один из постановщиков помех, то другой постановщик помех направляет раздвоенный помеховый луч на каждый истребитель. В промежутках времени, когда основной лепесток ДНА бортовой РЛС истребителя не облучает самолеты-постановщики помех, они включают свои БРЛС для уточнения местоположения подавляемых истребителей.

Таким образом, применение метода помехоустойчивости, основанного на подавлении боковых лепестков (ПБЛ), низкоэффективно при противодействии двум постановщикам помех, осуществляющим подавление бортовой РЛС по боковым лепесткам ДНА силовой, согласованной по спектру зондирующего сигнала помехи.

Альтернативным вариантом противодействия помехам, принимаемым по боковым лепесткам ДНА, является применение системы КБЛ, которая может быть реализована в том числе на АФАР, смысл которой заключается в формировании нулей ДНА на источник помехового воздействия. Однако недостатком данного подхода выступает, во-первых, низкая эффективность против импульсных помех, во-вторых, в случае постановки непрерывных помех постановщик активных помех может сопровождаться только по направлению, а дальность и скорость его полета измеряться не будут, в-третьих, может быть пропущена другая воздушная цель, направление на которую совпадает с направлением постановки помех.

Аналогичным образом может быть реализована и сигналоподобная силовая (превосходящая по мощности зондирующий сигнал) помеха по боковым лепесткам ДНА, направленная на внесение ложной информации в приемный канал, и, наряду с принципиальной возможностью извлекать радиолокационную информацию из радиолокационного сигнала в условиях помех такого типа, при реализации рассмотренных методов помехозащиты уровень информативности БРЛС в данной ситуации будет ниже возможного. Таким образом, целесообразно разработать дополнительные меры, позволяющие извлекать максимальное количество информации из радиолокационного сигнала в условиях воздействия сигналоподобных помех по боковым лепесткам ДНА типа DRFM, т.е. повысить ее помехоустойчивость.

С учетом этого целью статьи является разработка метода повышения помехоустойчивости импульсно-доплеровской БРЛС при воздействии силовой сигналоподобной помехи типа DRFM

$$
-805-
$$


по боковым лепесткам диаграммы направленности антенны, устройства, его реализующего, а также оценка характеристик данного устройства на основе моделирования.

\section{Физический смысл метода}

Физический смысл данного метода [10] рассмотрен на примере тактической ситуации, приведенной на рис. 2.

На рис. 2 приняты следующие обозначения:

$\mathrm{V}_{\text {ист }}$ - скорость самолета-истребителя, оборудованного импульсно-доплеровской БРЛС;

$\mathrm{V}_{\text {вц }}$ - скорость самолета воздушной цели;

$\mathrm{V}_{\text {пп }}$ - скорость самолета-постановщика помех, осуществляющего прикрытие воздушной цели;

$\mathrm{R}_{\text {пп }}$ - расстояние от самолета-истребителя до самолета-постановщика помех;

$\mathrm{R}_{\text {вц }}$ - расстояние от самолета-истребителя до воздушной цели;

$\mathrm{R}_{\text {бс }}$ - интервал по фронту между воздушной целью и самолетом-постановщиком помех;

$\alpha$ - угол между линией визирования и вектором скорости самолета-постановщика помех.

Самолету-истребителю, оборудованному импульсно-доплеровской (ИД) БРЛС, летящему со скоростью, к примеру, 280 м/с, в воздушном бою противодействуют два самолета противника, при этом в рассматриваемой тактической ситуации один из них - воздушная цель, летящая со скоростью, к примеру, 290 м/с, а другой - постановщик помех, летящий со скоростью, к примеру, 300 м/с; оба самолета находятся на интервале по фронту, который составляет порядка 7 км, и летят на встречу самолету-истребителю. Радиальные скорости сближения самолета-истребителя и пары самолетов, рассчитанные с учетом их взаимного расположения, составили 570 и $600 \mathrm{M} / \mathrm{c}$ соответственно.

Для наглядности процесс обзора БРЛС истребителя, постановки помехи, в приведенной тактической ситуации (рис. 2) и возможность ее идентификации условно разбиты на три этапа.

Первый этап. Импульсно-доплеровская БРЛС осуществляет сканирование воздушного пространства главным лучом ДНА с компенсационным каналом (рис. 4), причем коэффициент направленного действия основного лепестка ДНА основного канала БРЛС с учетом принятой длины волны БРЛС $\lambda=4 \times 10^{-2}$ м и площади антенны порядка 0,7 м² составляет $\mathrm{G}=5498$.

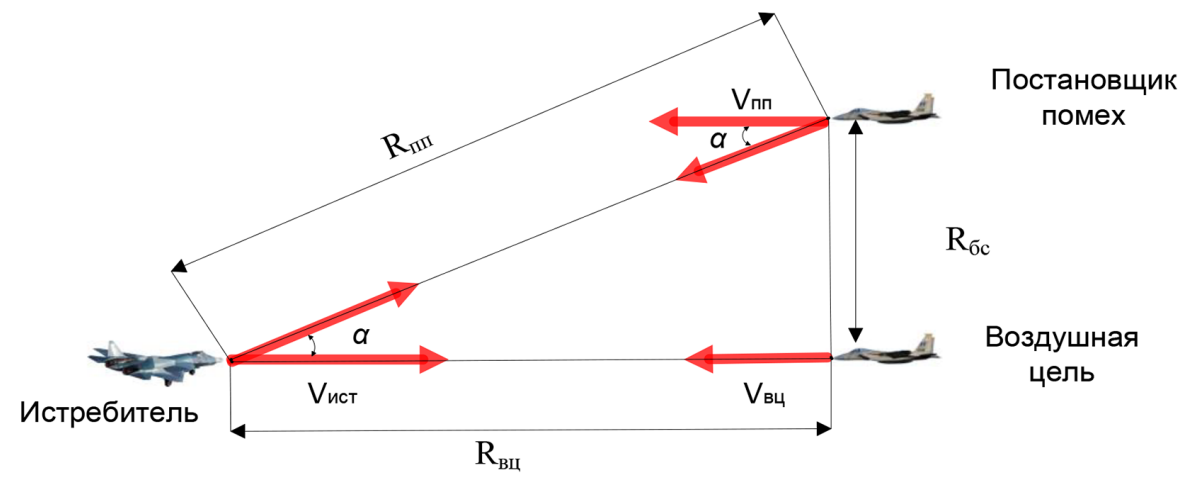

Рис. 2. Тактическая ситуация

Fig. 2. Tactical situation 


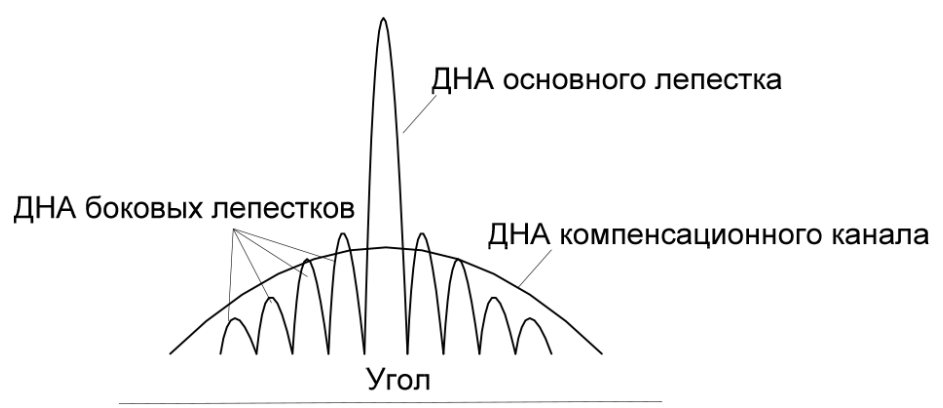

Рис. 3. Диаграмма направленности главной и компенсационной антенн

Fig. 3. The radiation pattern of the main and compensation antenna

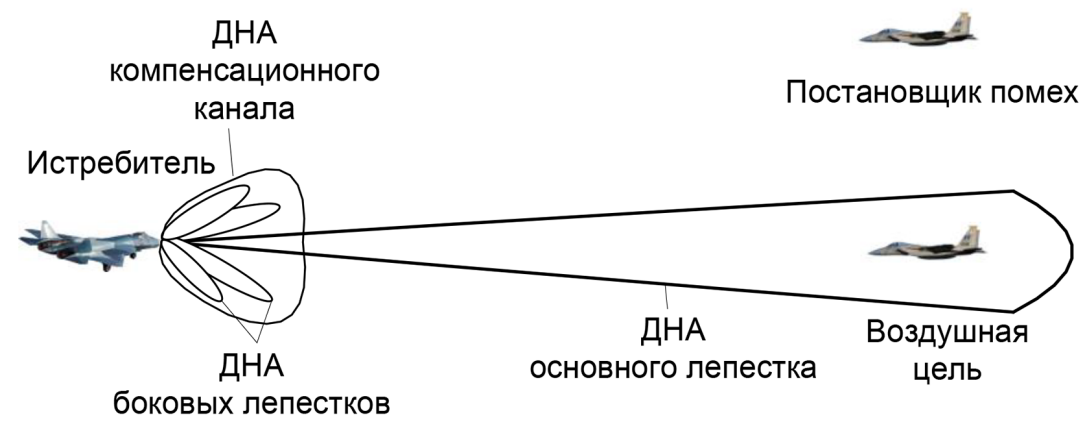

Рис. 4. Этап облучения главным лучом диаграммы направленности антенны импульсно-доплеровской бортовой радиолокационной станции самолета воздушной цели

Fig. 4. Stage of the main beam irradiation of the antenna pattern of a pulse-Doppler airborne radar station of a aircraft of an air target

Коэффициент направленного действия антенны компенсационного канала, как правило, выбирают таким образом, чтобы его значение незначительно превышало КНД боковых лепестков ДНА основного канала. Графическое представление соотношений КНД основного и компенсационного каналов, используемое на практике, представлено на рис. 3 [9].

Анализ рис. 3 свидетельствует о том, что на практике уровень боковых лепестков основного канала может незначительно превышать и принижать уровень компенсационного канала ввиду сложности точного формирования ДНА, незначительно превышающей все боковые лепестки основного канала.

При облучении самолета воздушной цели основной лепесток ДНА основного канала продолжает дальнейшее сканирование воздушного пространства, а принятый радиолокационный сигнал обрабатывается в двух каналах - в основном и компенсационном. Усиленный сигнал с учетом коэффициентов усиления основного и компенсационного каналов в каждом из данных каналов преобразуется с помощью процедуры быстрого преобразования Фурье в соответствующие амплитудно-частотные спектры. Амплитуды $\mathrm{A}_{1}$ и $\mathrm{A}_{2}$ (рис. 5а, б) спектральных составляющих сигнала соответственно в основном и компенсационном каналах с учетом подобранных коэффициентов усиления (КУ) в соответствующих каналах (КУ компенсационного канала не-

$$
-807-
$$




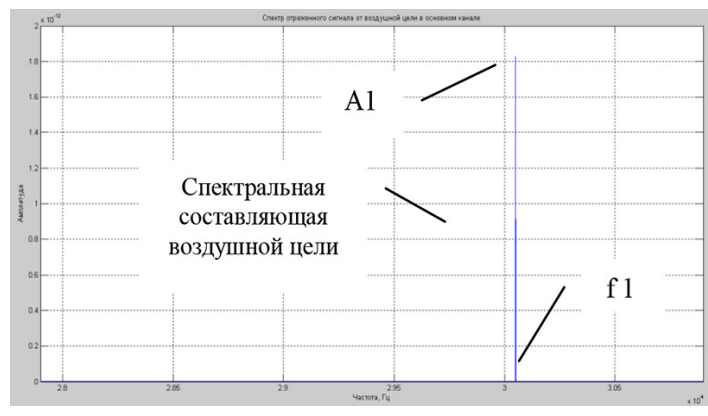

Рис. 5а. Спектр отраженного сигнала от самолета воздушной цели в основном канале

Fig. 5a. Spectrum of the reflected signal from an aircraft of an air target in the main channel

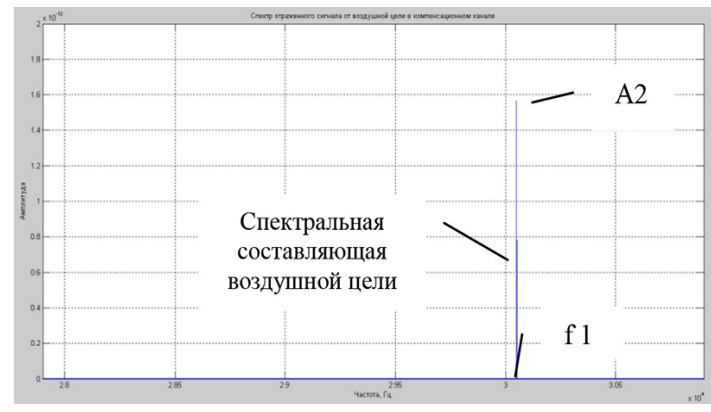

Рис. 5б. Спектр отраженного сигнала от самолета воздушной цели в компенсационном канале

Fig. 5б. Spectrum of the reflected signal from an aircraft of an air target in the compensation channel

значительно превышает КУ боковых лепестков основного канала) и эффективной поверхностью рассеивания (ЭПР) обнаруживаемой ВЦ расположены на частотной позиции $\mathrm{f}_{1}=30050$ Гц, обусловленной скоростью взаимного сближения истребителя и воздушной цели.

Расположение спектральных составляющих амплитудно-частотных спектров в основном и компенсационном каналах на частотной позиции $\mathrm{f}_{1}$ и выполнение условия (1) соответствуют отсутствию воздействия силовой сигналоподобной помехи типа DRFM по боковым лепесткам ДНА импульсно-доплеровской БРЛС, в этом случае осуществляется обработка только полезного сигнала в импульсно-доплеровской БРЛС.

$$
\mathrm{A}_{1}>\mathrm{A}_{2}
$$

Второй этап. В следующий момент времени, когда при облучении самолета-постановщика помех (рис. 6), оснащенного станцией радиотехнической разведки (РТР) и помех, главным лучом ДНА импульсно-доплеровской БРЛС, амплитуды $\mathrm{A}_{3}$ и $\mathrm{A}_{4}$ спектральных составляющих сигнала соответственно в основном и компенсационном каналах (рис. 7a, б), с учетом коэффициентов усиления в соответствующих каналах и ЭПР самолета-постановщика помех, расположены на частотной позиции $\mathrm{f}_{2}$, обусловленной доплеровским смещением несущей частоты импульсно-доплеровской БРЛС, вследствие взаимного перемещения ее носителя и облучаемого самолета-постановщика силовой сигналоподобной помехи типа DRFM. Если расположение спектральных составляющих в основном и компенсационном каналах амплитудно-частотных спектров на частотной позиции $\mathrm{f}_{2}$, которая составляет 28730 Гц, и выполнение условия (2) соответствуют отсутствию воздействия силовой сигналоподобной помехи типа DRFM по боковым лепесткам ДНА импульсно-доплеровской БРЛС, в этом случае осуществляется обработка только полезного сигнала в импульсно-доплеровской БРЛС. Самолет-постановщик помех, оборудованный станциями РТР и помех, в данный момент осуществляет определение и запоминание частоты зондирующего сигнала БРЛС.

$$
\mathrm{A}_{3}>\mathrm{A}_{4} .
$$

Третий этап. При облучении ВЦ главным лучом ДНА импульсно-доплеровской БРЛС (рис. 8) и постановке самолетом-постановщиком помех сигналоподобной помехи по боковым 


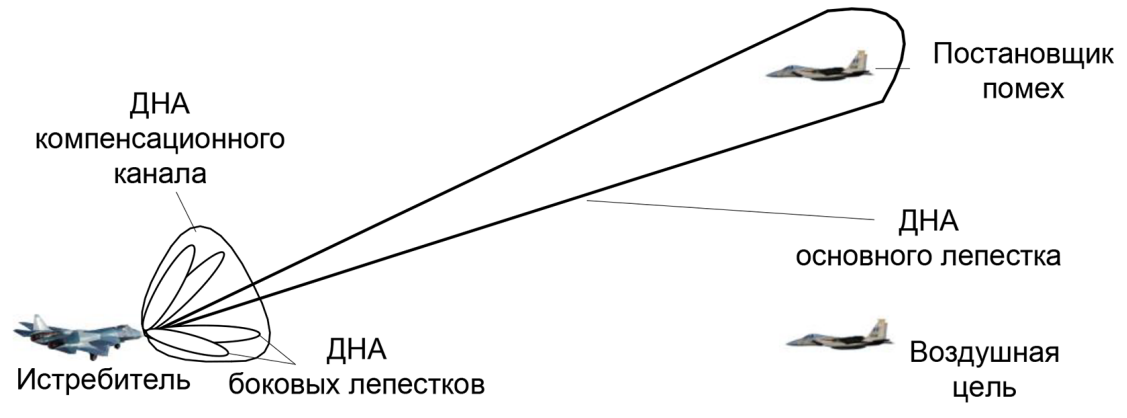

Рис. 6. Этап облучения главным лучом диаграммы направленности антенны импульсно-доплеровской бортовой радиолокационной станции самолета-постановщика помех

Fig. 6. Stage of the main beam irradiation of the antenna pattern of a pulse-Doppler airborne radar station of a aircraft of a jammer

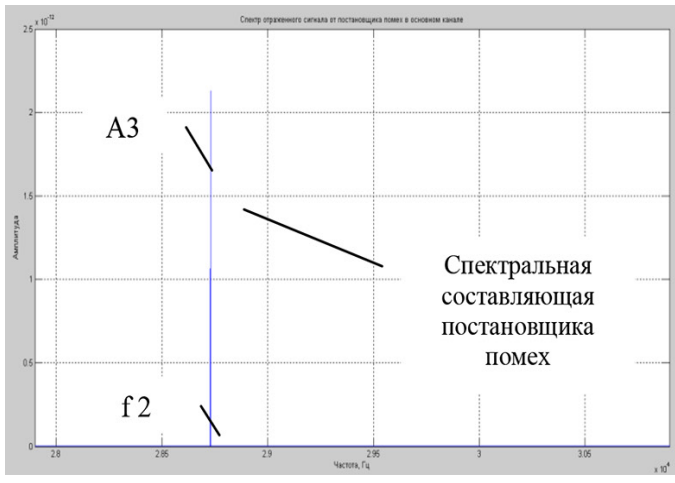

Рис. 7а. Спектр отраженного сигнала от самолетапостановщика помех в основном канале

Fig. $7 a$. Spectrum of the reflected signal from the aircraft of the jammer in main channel

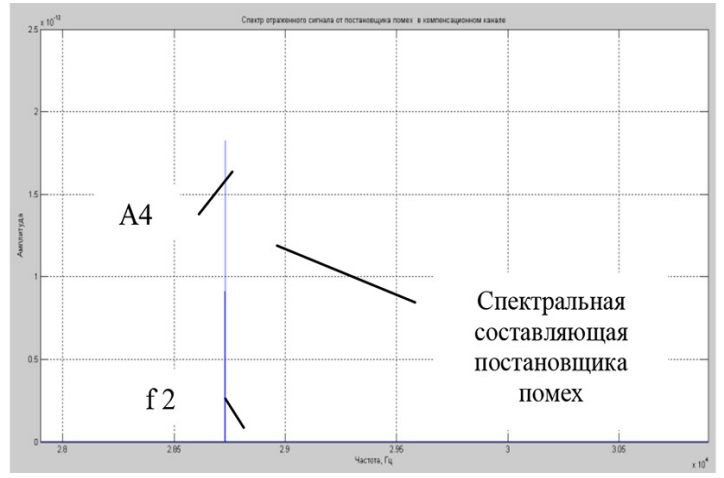

Рис. 7б. Спектр отраженного сигнала от самолетапостановщика помех в компенсационном канале

Fig. 76. Spectrum of the reflected signal from the aircraft of the jammer in compensation channel

лепесткам диаграммы направленности антенны на частотной позиции $\mathrm{f}_{2}$, которая была ранее разведана с помощью станции РТР, амплитуды $\mathrm{A}_{1}$ и $\mathrm{A}_{2}$ спектральных составляющих сигнала соответственно в основном и компенсационном каналах, с учетом коэффициентов усиления и ЭПР обнаруженной ВЦ, расположены на частотной позиции $\mathrm{f}_{2}$, амплитуды $\mathrm{A}_{\text {п1 }}$ и $\mathrm{A}_{\text {п2 }}$ спектральных составляющих помехового сигналоподобного сигнала соответственно в основном и компенсационном каналах, с учетом коэффициентов усиления в соответствующих каналах расположены на частотной позиции $\mathrm{f}_{1}$. В спектроанализаторе осуществляется анализ расположения спектральных составляющих сигнала и их амплитуд, при этом одновременное расположение спектральных составляющих сигнала (рис. 9 a, б) на частотных позициях $\mathrm{f}_{1}$ и $\mathrm{f}_{2}$ и одновременное выполнение условия (3) соответствует воздействию силовой сигналоподобной помехи типа DRFM по боковым лепесткам ДНА импульсно-доплеровской БРЛС; кроме того, имеется возможность определить частотную позицию $\mathrm{f}_{2}$, на которой организована силовая сигналоподобная помеха типа DRFM, с целью последующего ее исключения из дальнейшей обработки. 


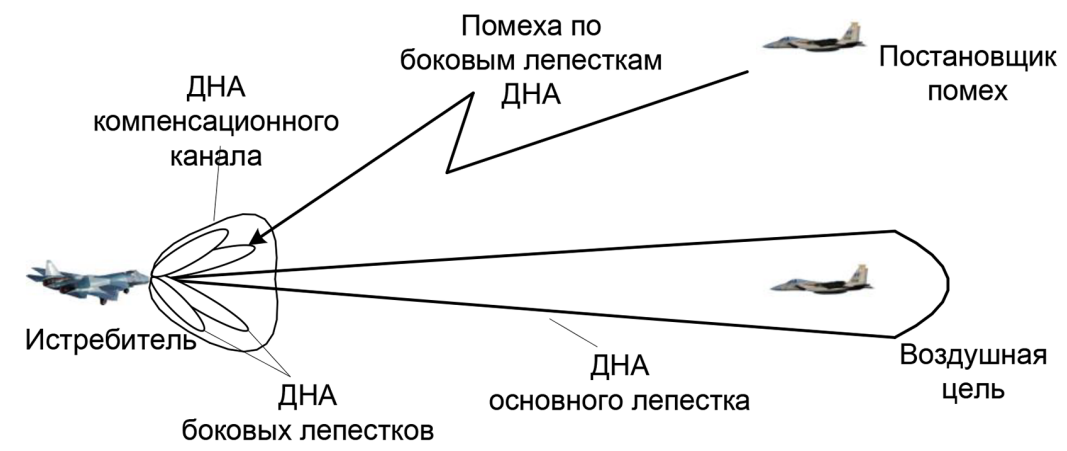

Рис. 8. Этап облучения главным лучом ДНА ИД БРЛС самолета воздушной цели и организации помехи по БЛ ДНА ИД БРЛС самолетом-постановщиком помех

Fig. 8. Stage of the main beam irradiation of the antenna pattern of a pulse-Doppler airborne radar station of an aircraft of a target and inherence along the side lobes of a pulsed Doppler radar station by a jamming aircraft

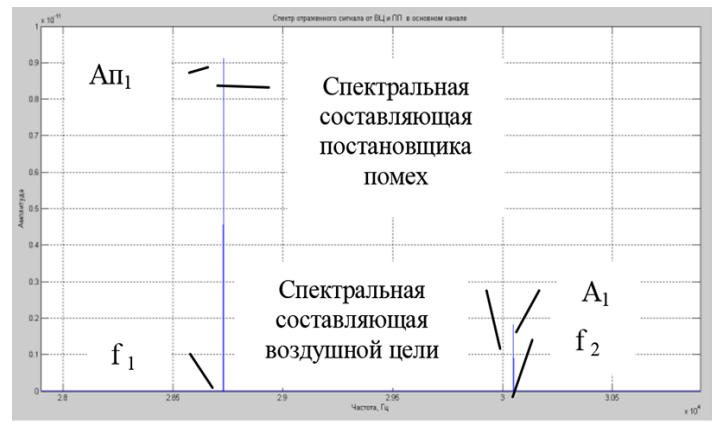

Рис. 9а. Спектр отраженного сигнала от воздушной цели и помехи от самолета-постановщика помех в основном канале

Fig. 9a. Spectrum of the reflected signal from an air tangent interference from an airplane

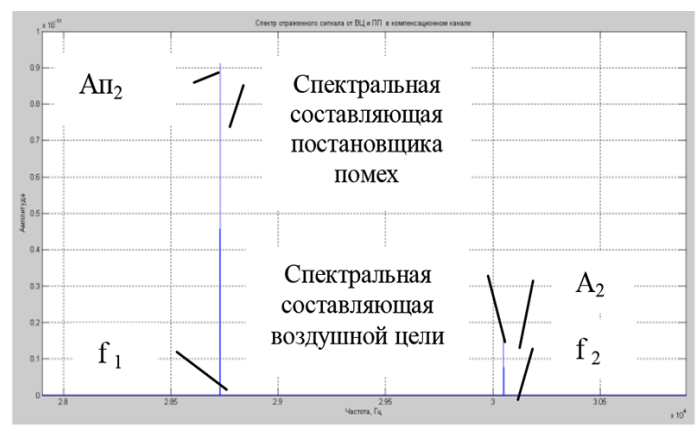

Рис. 9б. Спектр отраженного сигнала от воздушной цели и помехи от самолета-постановщика помех в компенсационном канале

Fig. 96. Spectrum of the reflected signal from an air tangent interference from aircraft of the jammer in the compensation channel

$$
\begin{aligned}
& A_{\Pi 1}>A_{1}, \\
& A_{\Pi 2}>A_{2}, \\
& A_{\Pi 2}>A_{\Pi 1} .
\end{aligned}
$$

Таким образом, разработанный метод позволяет на основе совместного спектрального анализа радиолокационного сигнала, принятого в основном и компенсационном каналах, идентифицировать воздействие помехи и осуществить дальнейшую его обработку с одновременной режекцией помехи.

\section{Устройство, реализующее метод}

На рис. 10 представлена техническая реализация метода повышения помехоустойчивости импульсно-доплеровской БРЛС при воздействии силовой сигналоподобной помехи типа DRFM по боковым лепесткам ДНА в виде устройства.

Принятые сигналы $\mathrm{S}_{\mathrm{o}}(\mathrm{t})$ и $\mathrm{S}_{\mathrm{k}}(\mathrm{t})$ в основном и компенсационном каналах с учетом коэффициентов усиления данных каналов и ЭПР поступают соответственно в приемники ПРМ1 
и ПРМ2. Из приемников сигналы $\mathrm{S}_{1}(\mathrm{t})$ и $\mathrm{S}_{2}(\mathrm{t})$ поступают на соответствующие блоки быстрого преобразования Фурье БПФ 3 и БПФ 4, где преобразуются в соответствующие амплитудночастотные спектры $\mathrm{S}_{1}(\mathrm{f})$ и $\mathrm{S}_{2}(\mathrm{f})$ основного и компенсационного каналов.

Далее в интересах идентификации помех, с целью ее последующего исключения, в спектроанализаторе CA5 осуществляется совместный анализ расположения составляющих амплитудно-частотных спектров $\mathrm{S}_{1}(\mathrm{f})$ и $\mathrm{S}_{2}(\mathrm{f})$ и их амплитуд.

При выполнении условий 1 или 2 , что соответствует отсутствию воздействия силовой сигналоподобной помехи типа DRFM, по боковым лепесткам ДНА импульсно-доплеровской БРЛС на выходе спектроанализатора СА5 формируется сигнал логического нуля «0», который является сигналом коммутатору 6 на распределение полезного сигнала $S_{1}(f)$ на первый выход импульсно-доплеровской БРЛС (рис. 10) для его последующей обработки. В случае выполнения условия 3, что соответствует воздействию силовой сигналоподобной помехи типа DRFM, по боковым лепесткам ДНА импульсно-доплеровской БРЛС на выходе спектроанализатора CA5 (рис. 10) формируется сигнал логической единицы «1», который служит сигналом коммутатоpy 6 на перераспределение сигнала с основного канала $\mathrm{S}_{1}(\mathrm{f})$ в перестраиваемый режекторный фильтр, на второй вход которого поступает значение частотной позиции, на которой располагается помеха $\mathrm{f}_{2}$.

В режекторном фильтре РФ7 спектральная составляющая помехи типа DRFM на частотной позиции $\mathrm{f}_{2}$ вырезается, и полезный сигнал $\mathrm{S}_{1}(\mathrm{f})$ поступает на второй выход импульсно-доплеровской БРЛС для его обработки.

\section{Оценка характеристик устройства на основе моделирования}

В интересах количественной оценки эффективности разработанного метода и устройства, его реализующего, было проведено имитационное моделирование. При моделировании были использованы следующие исходные данные:

Несущая частота зондирующего сигнала при выбранной длине волны $\lambda=4 \times 10^{-2}$ м составила $\lambda=7,5 \times 10^{9}$ Гц. Время когерентно-обрабатываемого сигнала $T_{\text {кн }}=10^{-3} \mathrm{c}$.

В качестве показателя эффективности работоспособности метода была определена вероятность правильной идентификации Р воздействия сигналоподобной помехи по боковым лепесткам ДНА антенны.

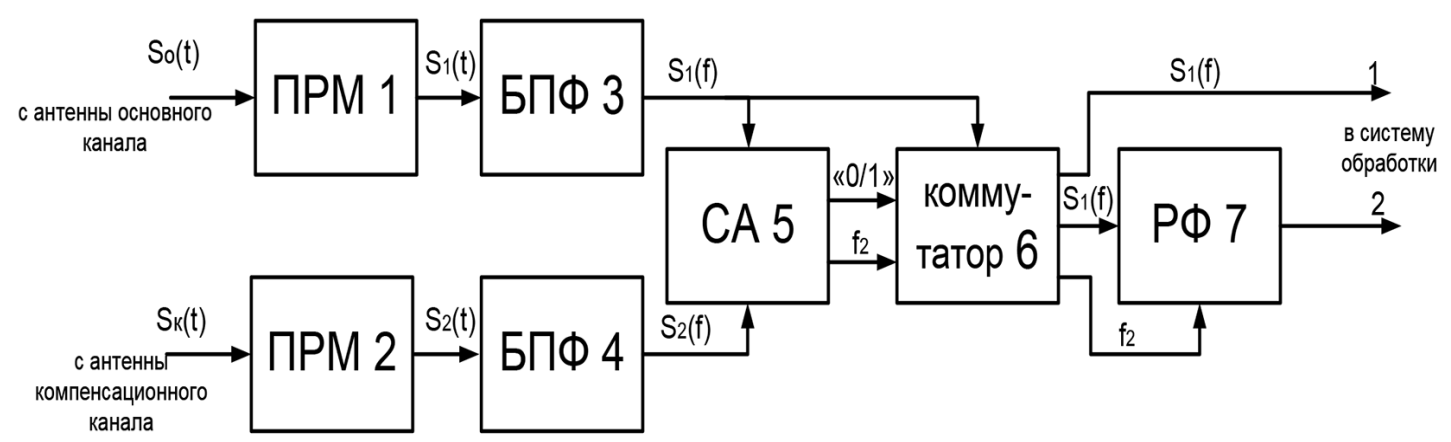

Рис. 10. Устройство, реализующее метод повышения помехоустойчивости

Fig. 10. Device implementing the noise immunity method 
Вследствие того, что решения о воздействии помехи принимают на основе сравнения амплитуд спектральных составляющих в основном и компенсационном каналах, в работе была выдвинута гипотеза, что основное влияние на эффективность работы разработанного метода оказывает соотношение коэффициентов усиления антенн основного и компенсационного каналов.

С учетом этого в интересах оценки вероятностных характеристик устройства, реализующего метод, был смоделирован процесс идентификации помехи при различных отношениях коэффициентов усиления компенсационного канала к коэффициенту усиления боковых лепестков ДНА основного канала $\mathrm{G}_{\mathrm{k}} / \mathrm{G}_{\mathrm{bl}}$. В предположении того, что значение мощности помехи, создаваемое противником, может быть различным, была оценена зависимость вероятности правильной идентификации от различных значений отношений мощности помехи к мощности отраженного сигнала с последующей статистической обработкой получаемых результатов. Результаты моделирования представлены на рис. 11-15 и в табл. 1.

Так, на рис. 11 приведена зависимость вероятности правильной идентификации сигналоподобной помехи типа DRFM от отношения мощности помехи к мощности сигнала $\mathrm{h}$ на входе антенн основного и компенсационного каналов, при отношении коэффициента усиления компенсационного канала к коэффициенту усиления боковых лепестков основного канала к $\mathrm{G}_{\mathrm{k}} / \mathrm{G}_{\mathrm{bl}}=0,9999$. Анализ данной зависимости свидетельствует о том, что при незначительном превышении мощности помехи мощности отраженного сигнала вероятность идентификации воздействия сигналоподобной помехи типа DRFM по боковым лепесткам ДНА незначительно увеличивалась. При превышении мощности помехи относительно мощности отраженного сигнала в диапазоне h от 1 до 1,4 вероятность правильной идентификации помехи составляет порядка 0,3. В дальнейшем по мере увеличения мощности помехи более чем в 3 раза вероятность идентификации помехи уменьшается и стремится к 0 .

Анализ зависимости вероятности правильной идентификации от отношения мощности помехи к мощности сигнала при $\mathrm{G}_{\mathrm{k}} / \mathrm{G}_{\mathrm{bl}}=1$, представленной на рис. 12 , показал, что макси-

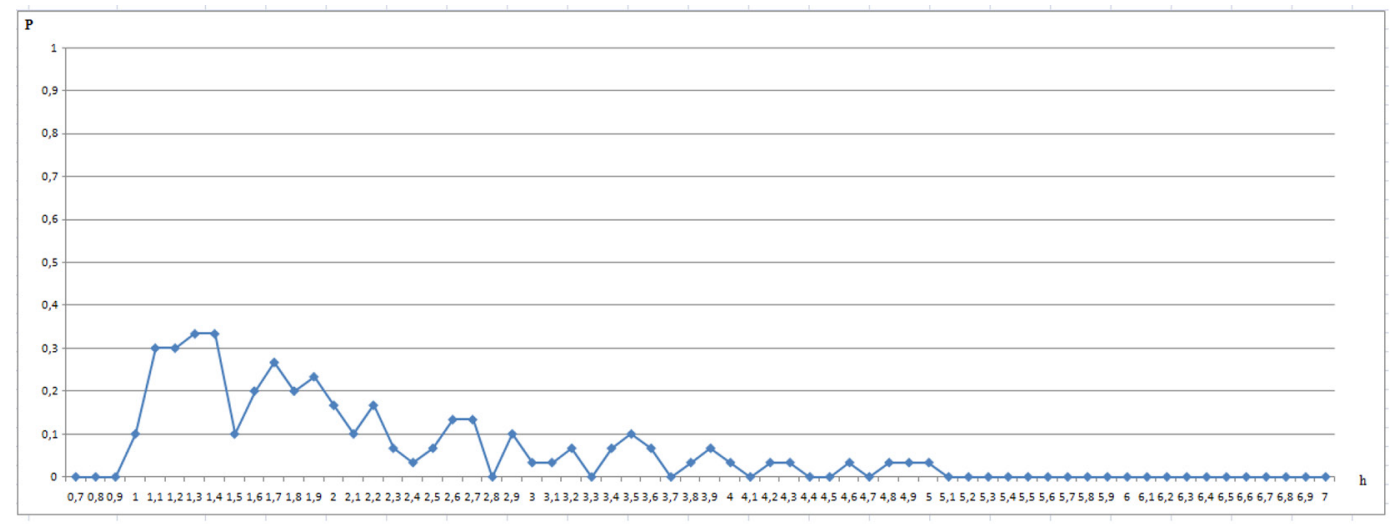

Рис. 11. График зависимости вероятности идентификации помехи при воздействии силовой сигналоподобной помехи по БЛ ДНА (при $\mathrm{G}_{\mathrm{k}} / \mathrm{G}_{\mathrm{bl}}=0,9999$ )

Fig. 11. The dependence of the probability of identification of interference when exposed to power signal-like interference along the side lobes of antenna radiation pattern $\left(\right.$ at $\left.G_{k} / G_{b l}=0,9999\right)$ 


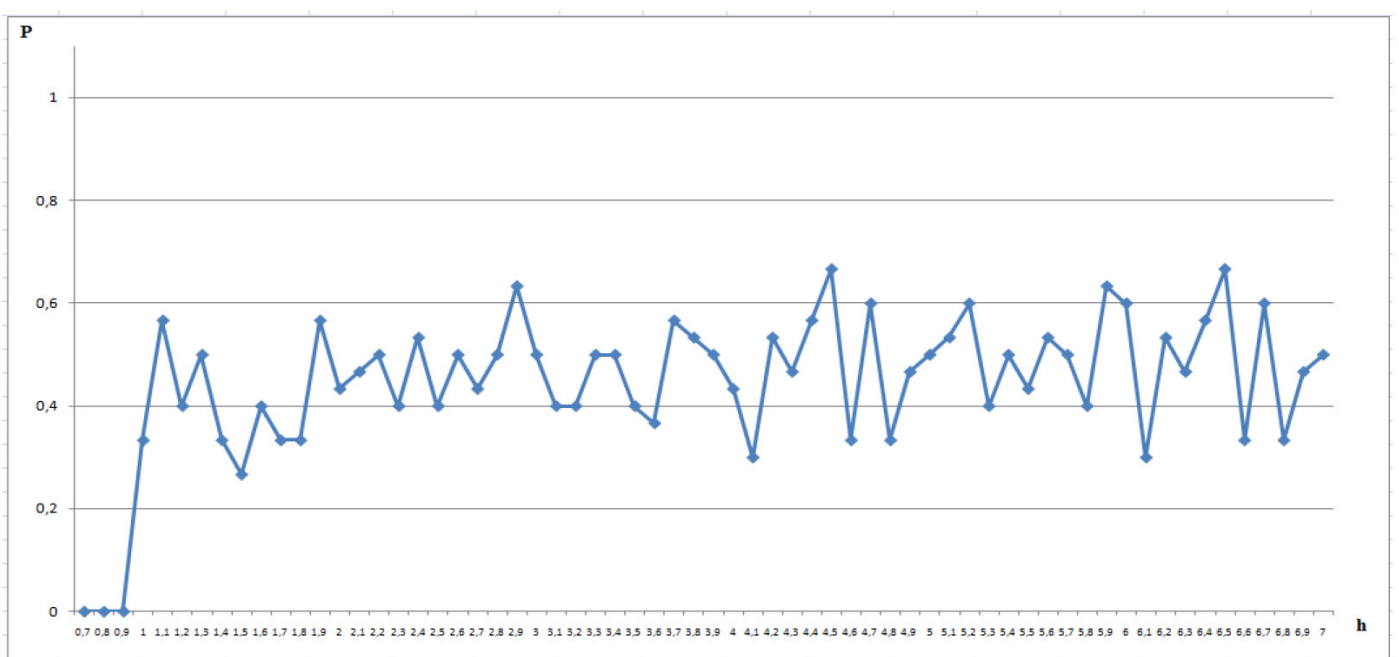

Рис. 12. График зависимости вероятности идентификации помехи при воздействии силовой сигналоподобной помехи по БЛ ДНА (при $\mathrm{G}_{\mathrm{k}} / \mathrm{G}_{\mathrm{bl}}=1$ )

Fig. 12. The dependence of the probability of identification of interference when exposed to power signal-like interference along the side lobes of antenna radiation pattern $\left(\right.$ at $\left.\mathrm{G}_{\mathrm{k}} / \mathrm{G}_{\mathrm{bl}}=1\right)$

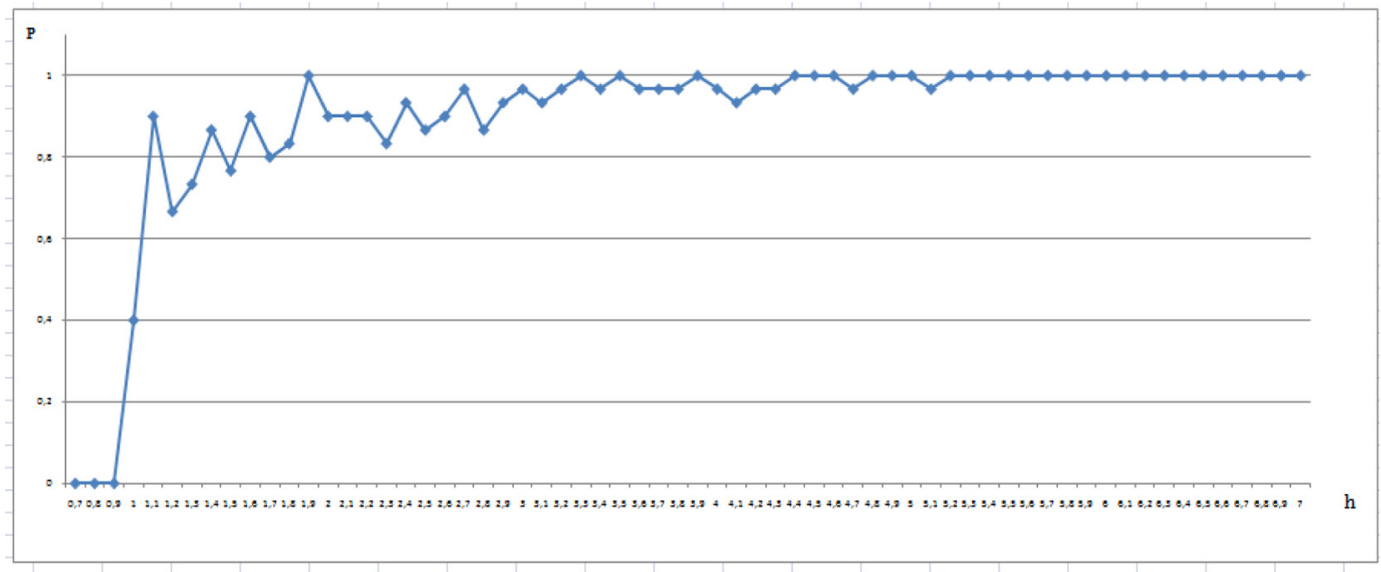

Рис. 13. График зависимости вероятности идентификации помехи при воздействии силовой сигналоподобной помехой по БЛ ДНА (при $\left.\mathrm{G}_{\mathrm{k}} / \mathrm{G}_{\mathrm{bl}}=1,001\right)$

Fig. 13. The dependence of the probability of identification of interference when exposed to power signal-like interference along the side lobes of antenna radiation pattern $\left(a t \mathrm{G}_{\mathrm{k}} / \mathrm{G}_{\mathrm{bl}}=1,001\right)$

мальное значение вероятности идентификации силовой сигналоподобной помехи типа DRM, воздействующей по боковым лепесткам ДНА, составила порядка 0,6 .

Анализ зависимости (рис. 13) вероятности правильной идентификации при $\mathrm{G}_{\mathrm{k}} / \mathrm{G}_{\mathrm{bl}}=1,0001$ показывает, что при увеличении мощности воздействия сигналоподобной помехи типа DRFM по боковым лепесткам ДНА вероятность правильной идентификации повышается и с превышением мощности помехи более чем в 2 раза вероятность правильной идентификации силовой сигналоподобной помехи стремится к 1. 
C дальнейшим увеличением отношения коэффициентов усиления $\mathrm{G}_{\mathrm{k}} / \mathrm{G}_{\mathrm{bl}}=1,0026$ и $\mathrm{G}_{\mathrm{k}} / \mathrm{G}_{\mathrm{bl}}=1,0052$ (рис. 14,15 ) вероятность правильной идентификации силовой сигналоподобной помехи повышается и при превышении мощности помехи в 1,1 раза мощности отраженного сигнала вероятность идентификации стремится к 1.

Совместный анализ результатов моделирования, приведенных на рис. 11-15, представлен в табл. 1. Анализ данных таблицы позволяет установить, что при превышении отношения ко-

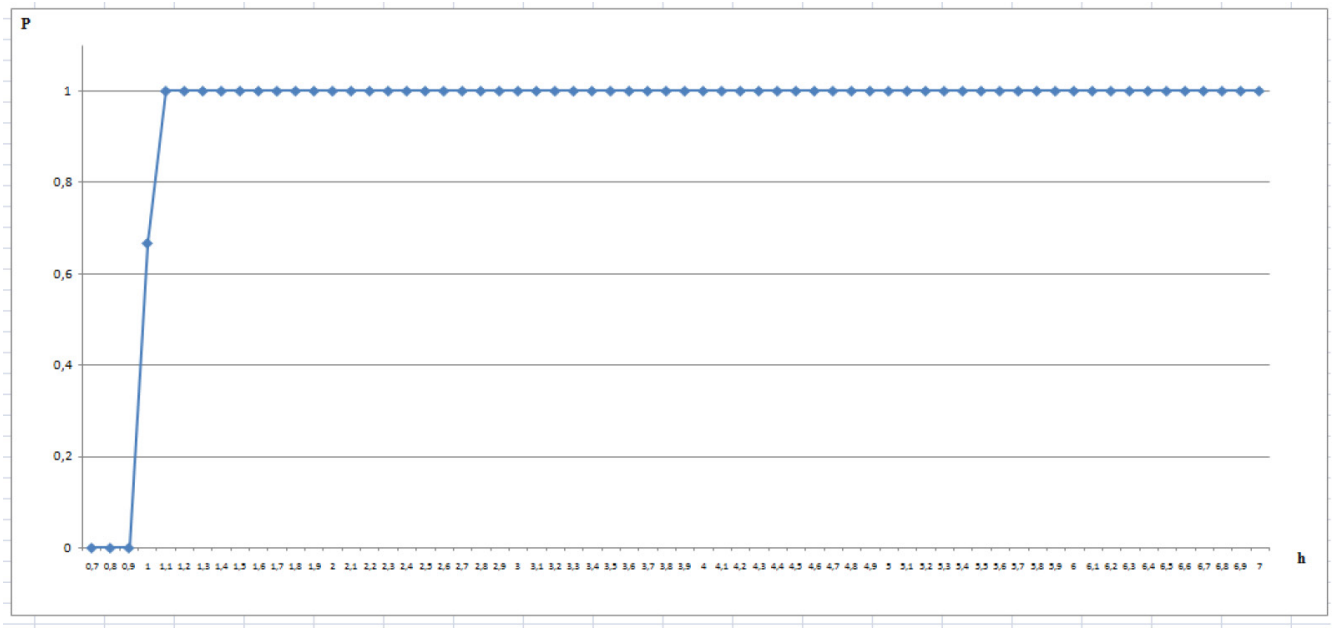

Рис. 14. График зависимости вероятности идентификации помехи при воздействии силовой сигналоподобной помехой по БЛ ДНА (при $\left.\mathrm{G}_{\mathrm{k}} / \mathrm{G}_{\mathrm{bl}}=1,0026\right)$

Fig. 14. The dependence of the probability of identification of interference when exposed to power signal-like interference along the side lobes of antenna radiation pattern $\left(\right.$ at $\left.\mathrm{G}_{\mathrm{k}} / \mathrm{G}_{\mathrm{bl}}=1,0026\right)$

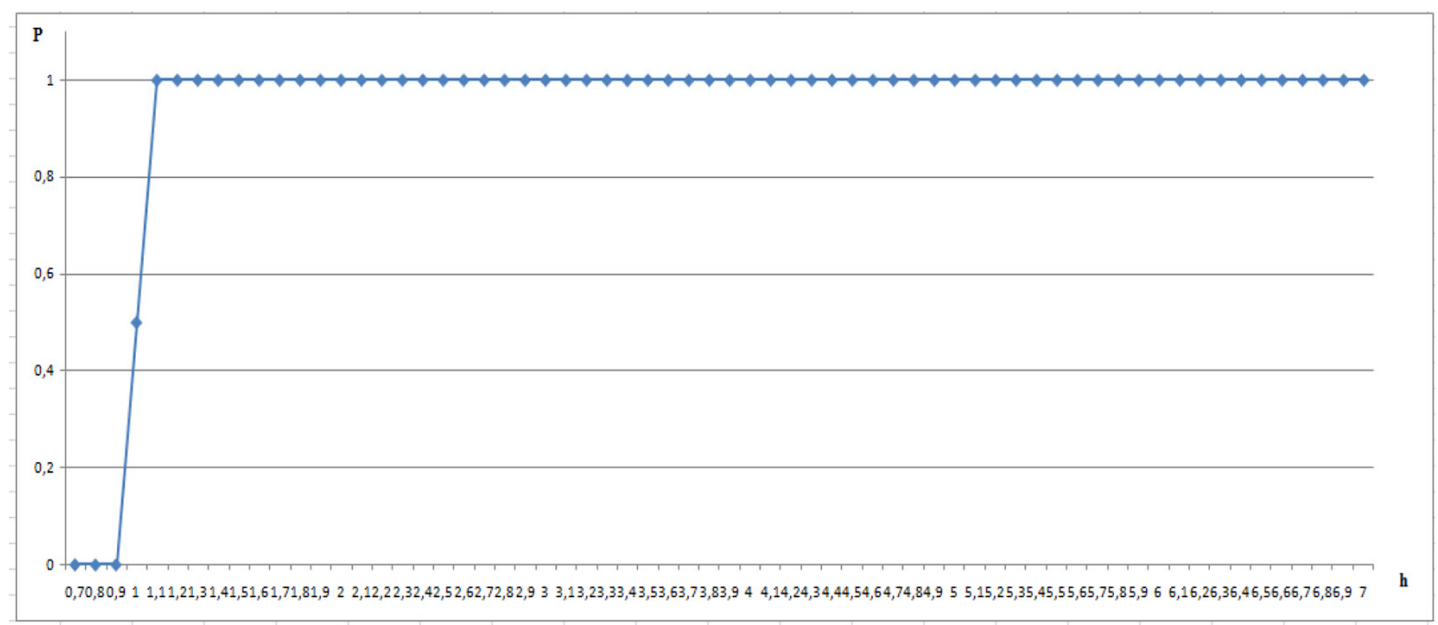

Рис. 15. График зависимости вероятности идентификации помехи при воздействии силовой сигналоподобной помехой по БЛ ДНА (при $\left.\mathrm{G}_{\mathrm{k}} / \mathrm{G}_{\mathrm{bl}}=1,0052\right)$

Fig. 15. The dependence of the probability of identification of interference when exposed to power signal-like interference along the side lobes of antenna radiation pattern $\left(\right.$ at $\left.G_{k} / G_{b l}=1,0052\right)$ 
Таблица 1. Таблица оценки результата моделирования

Table 1. Simulation result evaluation table

\begin{tabular}{|c|c|c|c|}
\hline \multirow{2}{*}{$\begin{array}{l}\text { Соотношения } \\
\text { коэффициентов } \\
\text { усиления } \mathrm{Gk} / \mathrm{Gbl}\end{array}$} & \multicolumn{3}{|c|}{$\begin{array}{c}\text { Вероятность идентификации силовой сигналоподобной помехи типа DRFM, } \\
\text { действующей по боковым лепесткам ДНА метода } \\
\text { повышения помехоустойчивости ИД БРЛС }\end{array}$} \\
\hline & $\begin{array}{c}\text { Диапазон изменения } \\
\text { мощности помехи } \\
\mathrm{h}=0,7-0,9\end{array}$ & $\begin{array}{c}\text { Диапазон изменения } \\
\text { мощности помехи } \\
\mathrm{h}=0,9-1,1\end{array}$ & $\begin{array}{l}\text { Диапазон изменения } \\
\text { мощности помехи } \\
\mathrm{h}=1,1-7\end{array}$ \\
\hline 0,9999 & $\rightarrow 0$ & $0,1 \rightarrow 0,99$ & $\begin{array}{c}0,03-0,33 \text { (при hот 1,1 до 5) } \\
\rightarrow 0 \text { (при } \mathrm{h}>5)\end{array}$ \\
\hline 1 & $\rightarrow 0$ & $0,1 \rightarrow 0,99$ & $0,13-0,66$ \\
\hline 1,001 & $\rightarrow 0$ & $0,1 \rightarrow 0,99$ & $\begin{array}{c}\text { 0,63-1 (при hот } 1,1 \text { до 5) } \\
\rightarrow 1 \text { (при } \mathrm{h}>5)\end{array}$ \\
\hline 1,0026 & $\rightarrow 0$ & $0,1 \rightarrow 0,99$ & $\rightarrow 1$ \\
\hline 1,0052 & $\rightarrow 0$ & $0,1 \rightarrow 0,99$ & $\rightarrow 1$ \\
\hline
\end{tabular}

эффициента усиления компенсационного канала к коэффициенту усиления основного канала величины $\mathrm{G}_{\mathrm{k}} / \mathrm{G}_{\mathrm{bl}}=1,0026$ вероятностные характеристики устройства, реализующего метод, соответствуют приемлемым на практике требованиям при всех диапазонах соотношений помеха/сигнал.

\section{Выводы}

Таким образом, устройство, реализующее разработанный метод, позволит:

1. Устранить выявленные ранее недостатки в методах работы системы ПБЛ и КБЛ при воздействии сигналоподобной помехи типа DRFM по боковым лепесткам ДНА, обеспечив устойчивое функционирование ИД БРЛС как при импульсных, так и при непрерывных помехах по боковым лепесткам ДНА.

2. Расширить функциональные возможности импульсно-доплеровской БРЛС за счет распознавания воздействия по боковым лепесткам ДНА силовой сигналоподобной помехи типа DRFM при обнаружении и сопровождении воздушной цели, прикрываемой самолетомпостановщиком помех.

3. Оценка характеристик устройства, реализующего разработанный метод, позволила установить, что при выборе соотношения коэффициентов усиления $\mathrm{G}_{\mathrm{k}} / \mathrm{G}_{\mathrm{bl}}=1,0026$ обеспечиваются приемлемые на практике вероятностные характеристики идентификации помехи.

\section{Список литературы / References}

[1] Осин В.А. Применение сил и средств РЭБ в локальных войнах и вооруженных конфликтах. Радиоэлектронная борьба в ВС РФ: тематический сб. - 2013. М., 2013. [Osin V.A. The use of forces and means of electronic warfare in local wars and armed conflicts. Electronic warfare in the Armed forces of the Russian Federation: thematic collection. - 2013. M., 2013 (in Russian)]

[2] Осин В.А. Применение сил и средств РЭБ в локальных войнах и вооруженных конфликтах. Радиоэлектронная борьба в ВС РФ: тематический сб. - 2014. М., 2014. [Osin V.A. The use 
of forces and means of electronic warfare in local wars and armed conflicts. Electronic warfare in the Armed forces of the Russian Federation: thematic collection. - 2014. M., 2014 (in Russian)]

[3] Осин В.А. Применение сил и средств РЭБ в локальных войнах и вооруженных конфликтах. Радиоэлектронная борьба в ВС РФ: тематический сб. - 2015. M., 2015. [Osin V.A. The use of forces and means of electronic warfare in local wars and armed conflicts. Electronic warfare in the Armed forces of the Russian Federation: thematic collection. - 2015. M., 2015 (in Russian)]

[4] Метод повышения помехозащищенности бортовой радиолокационной станции истребителя: монография. А.В. Богданов [и др.], рук. авт. коллектива Д.В. Закомолдин. Красноярск: Сиб. федер. ун-т, 2018. 182 с. [The method of increasing the noise immunity on-board fighter radar: monograph A.V. Bogdanov [and others], the head of copyright. collective D.V. Zakomoldin. Krasnoyarsk: Siberian Federal University, 2018. 182 p. (in Russian)]

[5] Помехозащита радиоэлектронных систем управления летательными аппаратами и оружием. В.Н. Лепин, В.Н. Антипов, А.Ю. Викентьев и др. М.: Радиотехника, 2017. [The method of increasing the noise immunity on-board fighter radar. V.N. Lepin, V.N. Antipov, A.U. Vikentev. M., Radiotehnika, 2017 (in Russian)]

[6] Ван Брант Л.Б. Справочник по методам радиоэлектронного подавления и помехозащиты систем с радиолокационным управлением оружием. Т. I, кн. III. M., 1985. [Van Brant L.B. Reference book on methods of electronic suppression and noise immunity of systems with radar weapon control. Vol. I, book III. M., 1985 (in Russian)]

[7] Пат. 1840239, МПК G01S7/36 2006.01, Способ подавления помех в области боковых лепестков антенны радиолокатора и устройство для его реализации. Б.Г. Беляев, Э.М. Соколова. № 4528815/09, заявл. 26.03.1990, опубл. 20.08.2006. [Patent. 1840239, IPC 001 \$7/36?? 2006.01, A method for suppressing interference in the region of the side lobes of a radar antenna and a device for its implementation. B.G. Belyaev, E.M. Sokolova. No. 4528815/09, statement 03/26/1990, published 08/20/2006 (in Russian)]

[8] Слатин В.В. Современные и перспективные авиатехнологии РЭБ и бортовые средства их реализации. Новости зарубежной науки и техники. Серия: Авиационные системы. М.: ГосНИИАС, 2016, 11, 15. [Slatin V.V. Modern and perspective aviation technologies of electronic warfare and airborne means of their implementation. News of foreign science and technology. Series: Aviation Systems. M.: GosNIIAS, 2016, 11, 15 (in Russian)]

[9] Сколник М.И. Справочник по радиолокации. В 2 кн., кн. 1. М.: Техносфера, 2014. [Skolnik M.I. Reference for radar. In 2 book, I. M.: Technosphere, 2014 (in Russian)]

[10] Богданов А.В., Васильев О.В., Голубенко В.А., Закомолдин Д.В., Каневский М.И., Кочетов И.В., Кучин А.А., Часовских С.А. Способ функционирования импульсно-доплеровской бортовой радиолокационной станции с распознаванием воздействия помехи из вынесенной точки пространства при обнаружении воздушной цели, прикрываемой постановщиком помех. Патент на изобретение № 2688188, 2019. .[Bogdanov A.V., Vasiliev O.V., Golubenko V.A., Zakomoldin D.V., Anevsky M.I., Kochetov I.V., Kuchin A.A., Chasovskikh S.A. The operation method of a pulse-Doppler airborne radar station with recognition of the effect of interference from a remote point in space when an aerial target is covered by an interference director. Patent for invention № 2688188, 2019 (in Russian)] 
[11] Wang X.U., Zhou Y.W. Wang Y.Y. An Improved Antenna Array Pattern Synthesis Method Using Fast Fourier Transforms. Advances in Antenna Array Processing for Radar 2014.

[12] Хьюз М. Основы антенны: диаграммы излучения, диэлектрическая проницаемость, направленность и усиление. Техническая статья. 2016 [Hughes M.H. Antenna Basics: Radiation Patterns, Permittivity, Directivity, and Gain. Technical article. 2016 (in Russian)]

[13] Новиков А.В., Хлусов В.А. Способ формирования диаграммы направленности антенной решетки на прием с низким уровнем боковых лепестков. Доклады Томского государственного университета систем управления и радиоэлектроники. Томск: ТГУ, 2017, 20, 2, 5-9. [Novikov A.V., Hlusov V.A. Method of forming a diagram antenna array for reception with a low level of side lobes. Reports of the Tomsk state University of control systems and Radioelectronics. Tomsk, 2017, 20, 2, 5-9 (in Russian)]

[14] Защита радиолокационных систем от помех. Состояние и тенденции развития. Под ред. А.И. Канащенкова и В.И. Меркулова. М.: Радиотехника, 2003. 416 с. [Protection of radar systems from interference. State and trends of development. Ed. by A.I. Kanashchenkov and V.I. Merkulov. M.: Radio engineering, 2003. 416 c. (in Russian)]

[15] Добыкин В.Д., Куприянов А.И., Пономарев В.Г., Шустов Л.Н. Радиоэлектронная борьба. Силовое поражение радиоэлектронных систем. Под ред. А.И. Куприянова. М.: Вузовская книга, 2007. 468 с. [Dobykin V.D., Kupriyanov A.I., Ponomarev V.G., Shustov L.N. Electronic warefare Power defeat of radio-electronic systems. Ed. by. A.I. Kupriyanov. M.: University book, 2007. 448 c. (in Russian)] 